\title{
Abstentions in the German Bundesrat and ternary decision rules in weighted voting systems
}

\author{
Olga Birkmeier, Andreas Käufl, Friedrich Pukelsheim
}

Received: April 17, 2010; Accepted: October 27, 2010

\begin{abstract}
Summary: Ternary decision rules allowing for abstentions, besides Yea- and Nay-votes, are analyzed. The German Bundesrat serves as a prime example. We show that the decision-making efficiency of the Bundesrat would increase if abstentions were allowed. A general formula for the mean success margin of a ternary decision rule is derived assuming selfdual and permutationally invariant distributions. The ternary Penrose-Banzhaf model is discussed in detail, and the influence probabilities of voters and a rule's influence sensitivity are evaluated.
\end{abstract}

\section{Introduction}

Many studies on decision rules concern models where voters are faced with just two options, to vote either Yea or Nay. In practical situations voters often have the third option of abstaining. An interesting example is provided by the German Bundesrat, the second chamber representing the sixteen German States. The Bundesrat Rules and Regulations do not provide for abstentions, yet States do abstain. Section 2 describes the specific Bundesrat procedure in greater detail. The remaining sections develop a probabilistic analysis of ternary decision rules.

The Council of Ministers of the European Union follows an unusual practice. Abstentions are mostly counted as Nay-votes. But they are taken to be Yea-votes when the adoption of a proposal requires unanimity, see Felsenthal-Machover (1998, page 335), Lindner (2004, page 23). There is a historical precursor for such flexibility. The 1802 Swiss Notablenverfassung (Constitution of the Notables) drew more Nay-votes than Yeavotes from the people. By classifying abstentions as Yeas, the Notables manufactured a majority and the constitution entered into force (Reinhardt 2006, page 89).

There are decision-making systems that respect the particular quality of abstentions. For instance, the notion of a straight majority may be realized in two ways. The first way

AMS 2000 subject classification: Primary: 90A28

Key words and phrases: Decision-making efficiency, Penrose-Banzhaf index, straight majority rule, unanimity rule, voting power, voting profiles 
requires more than half of the plenum to vote Yea. This implies that those who do not vote Yea are counted as opposers, whether they vote Nay or abstain. The second way requires more Yea-votes than Nay-votes. Voters who abstain are acknowledged to be undecided, and are not included in the comparison of Yea- and Nay-votes.

While much of the literature on decision rules focuses on the binary case of Yea-Nay voting, the analysis of ternary decision rules has now and then received some attention, see Felsenthal and Machover (2001) or Section 10.1 in Felsenthal and Machover (2005). The earliest settings, such as Rubinstein (1980, 2002) and Bolger (1983), consider more than two options the voters can choose, that is, there is an (unordered) multitude of levels how voters can express their choice. Fishburn (1973) also admits a third level of indifference, which may be interpreted as a way of abstention, though the thrust of his work wanders off in a different direction.

Later settings investigate an ordered set of ways how voters may express their choice, such as three levels of increasing consent ranging from Nay over abstention to Yea. This is the predominant view in Felsenthal and Machover (1997, 1998), Braham and Steffen (2002), Côrte-Real and Pereira (2004), and Lindner (2004). In particular, Freixas and Zwicker $(2003,2009)$ propose a systematic discussion how abstentions may be worked into the more classical binary decision rule theory. In their terminology, the present paper would be categorized as dealing with a $(3,2)$ voting game with ordered input alternatives, that is, voters have 3 ordered input levels to express themselves (Nay, abstention, Yea) and the resulting decision may attain 2 possible output levels (positive, negative).

The papers referred to focus on the game-analytic properties of multi-level decision rules, and their combinatorial structure. In the present paper we prefer a more probabilistically oriented viewpoint. The companion paper Ruff and Pukelsheim (2010) presents a probabilistic synopsis of binary decision rules, the present paper extends this approach to ternary decision rules. Straffin was among the first to rigorously follow up on a probabilistic view of the problem, see Straffin (1988) and the references given therein.

Our basic assumption is that all participants share a common probability $t$ of abstaining. For an a priori analysis we find this supposition to be quite reasonable. Theoretically, the abstention probability $t$ is taken to range from zero to unity. Practically, an abstention probability exceeding one third would seem to be of little real value. Felsenthal and Machover $(1997,1998)$ examine the case for $t=1 / 3$, that is, equal probabilities of voting Yea, Nay, or of abstaining. In the terminology of Freixas and Zwicker (2003), the type of weighted voting games that we use is ratio-weighted voting, as opposed to difference-weighted voting. Our motivation was to stay close to the practical needs of the current Bundesrat discussion.

Generally, abstentions could be taken as yet another level of approval besides Yea and Nay, and the probabilistic analysis may be reconciled with a more graph-theoretic approach. The interested reader is referred to the recent work of Freixas (2005a, b), Freixas and Palacios (2010), or Laruelle and Valenciano (2005, 2010a, b).

A brief overview of the paper is as follows. Section 2 presents a case study of some current interest, the German Bundesrat. Section 3 introduces our reference space, the set of ternary voting profiles, within which we can conveniently define the events of interest. Section 4 adds the probabilistic assumptions, with an emphasis on distributions that are selfdual and permutationally invariant. Section 5 applies the general theory to a specific 
model, the ternary Penrose-Banzhaf model. Section 6 adds a brief discussion of other modeling assumptions, and concludes with some final remarks.

\section{Abstentions in the German Bundesrat}

The Bundesrat (Federal Council) is one of the five constitutional organs of Germany, along with the Bundestag (Federal Diet, Parliament), Bundesregierung (Federal Government), Bundespräsident (Federal President) and Bundesverfassungsgericht (Federal Constitutional Court). The Bundesrat, the second chamber, is the assembly of the sixteen States of the Federal Republic of Germany.

Every State is allocated a certain number of Bundesrat seats. Delegation sizes only vaguely mirror population sizes, yet this has never been a point of concern. Four States send 6 delegates (Bavaria, Baden-Württemberg, Lower Saxony, North Rhine-Westphalia), one State 5 (Hesse), seven States 4 (Berlin, Brandenburg, Rhineland-Palatinate, Saxony, Saxony-Anhalt, Schleswig-Holstein, Thuringia), four States 3 (Bremen, Hamburg, Mecklenburg-Western Pomerania, Saarland). Thus the Bundesrat plenum consists of 69 members.

A State's delegation must submit their votes en bloc. In practice the head of delegation, usually the State's Prime Minister, carries the corresponding voting weight. Factually, then, four States command a voting weight of 6 units, one State voting weight 5, seven States voting weight 4 , and four States voting weight 3.

The Bundesrat uses two quotas. Normally, decisions call for a straight majority of more than half of the votes, so the one-half quota equals 35. Others, such as constitutional amendments, must reach at least a two-thirds quota, that is, 46 votes.

German State Governments are mostly coalition governments, with many of them composed of political parties other than those that constitute the Federal Government. Since quite a few proposals originate from the Federal Government and its administration, it occasionally happens that the coalition partners in a State cannot agree on a common vote. But their Bundesrat delegation must submit a bloc vote. So the consensus is to abstain, often laid down in the coalition manifesto for the legislative period. In this way the coalition governments at the state level shield themselves from being forced into a political fight that is triggered on the federal level and that is not under the State's control.

However, the Bundesrat Rules and Regulations do not provide for abstentions. Only Yea-votes count toward reaching the quotas of 35 or 46 . Thus the political practice is a bit peculiar. While the minutes do record whether a State abstains, the presidency only counts Yea-votes and does not distinguish between Nay-votes and abstentions.

With an increasing diversification of the political spectrum it nowadays happens more often than in former times that on the state level governments are formed by different political coalitions than on the federal level. This increases the pressure on the decisionmaking process in the Bundesrat in that it becomes increasingly harder to satisfy the quota.

For this reason Wolfgang Schäuble, Minister for Interior Affairs during the legislative period 2005-2009, proposed an amendment of the Bundesrat Rules and Regulations. Instead of referring the one-half and two-thirds quotas to the total number of votes (69), he 
proposed to refer them to the total number of Yea- and Nay-votes and thus to give abstentions a novel, neutralizing effect. Schäuble's intention was to strengthen the Bundesrat's decision-making efficiency, and to overcome its increasingly frequent stalemates.

The present paper quantifies the effects of abstentions in the Bundesrat procedures. As a measure for its decision-making capability we calculate the efficiencies of the Bundesrat decision rules. We also investigate how abstentions affect the influence probabilities and the power shares of the States, though this issue was not addressed in Schäuble's proposal.

We adopt the ternary Penrose-Banzhaf model. Voters abstain with probability $t$, while they vote Yea or Nay with half of the remaining probability, $(1-t) / 2$. The minutes of the Bundesrat confirm that States vote more often Yea or Nay than abstain. Unfortunately we did not succeed in acquiring sufficient empirical data from the Bundesrat archives in order to differentiate between the voting behavior of the various States. Of course, it is a bold hypothesis to assume the same abstention probability for all States. On the other hand, from an a priori point of view, common abstention probabilities appear to be a natural starting point for a first study. We focus on abstention probabilities between 0 and $1 / 3$.

We evaluate the indices of interest by treating abstentions either as pseudo abstentions, or as true abstentions:

- Pseudo abstentions: Abstentions are counted as Nay-votes. The percentage quota is evaluated statically, relative to the sum of the weights of all votes (69).

- True abstentions: Abstentions are recorded as a category of their own, besides Yeaand Nay-votes. The one-half and two-thirds quotas are evaluated dynamically, relative to the sum of the weights of Yea- and Nay-votes.

The results of our ternary analysis are summarized in Figures 2.1 and 2.2.

Decision-making efficiency, current pseudo abstentions

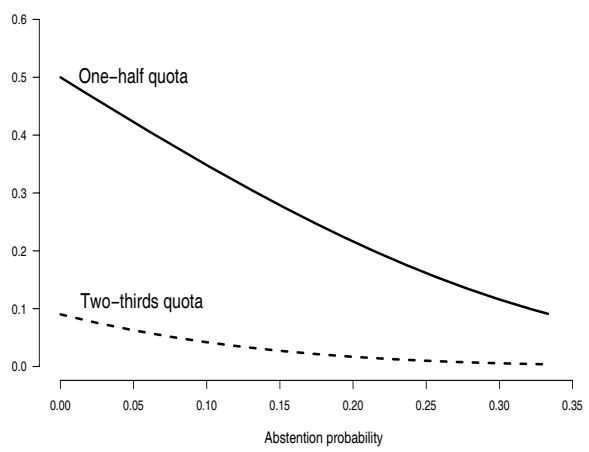

Decision-making efficiency, hypothetical true abstentions

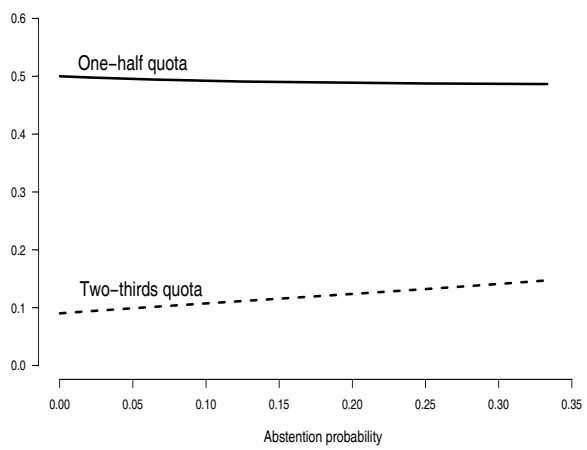

Figure 2.1 Decision-making efficiencies in the Bundesrat. Under current pseudo abstentions, efficiencies diminish when the abstention probability grows larger (left panel). Under hypothetical true abstentions, efficiencies are unaffected for the one-half quota, and slightly increase for the two-thirds quota (right panel).

Figure 2.1 exhibits the behavior of the decision-making efficiency of the decision rules when the abstention probability $t$ increases from 0 to $1 / 3$. The efficiency index tells 
how likely a random voting profile turns out to be positive and leads to the acceptance of the proposal. The top lines relate to a quota of one-half, the bottom lines of two-thirds.

The left panel reflects current conditions, pseudo abstentions. The decision-making efficiency of the Bundesrat is seen to decrease, very much so in the case of the one-half quota, and just a little bit for the two-thirds quota.

The right panel displays the behavior in the case of true abstentions. True abstentions have almost no effect on the one-half quota and leave the decision-making efficiency virtually constant. In the case of the two-thirds quota the efficiencies are even slightly increasing. Figure 1 confirms the intuitive judgment of Wolfgang Schäuble that by acknowledging true abstentions the Bundesrat would operate more efficiently.
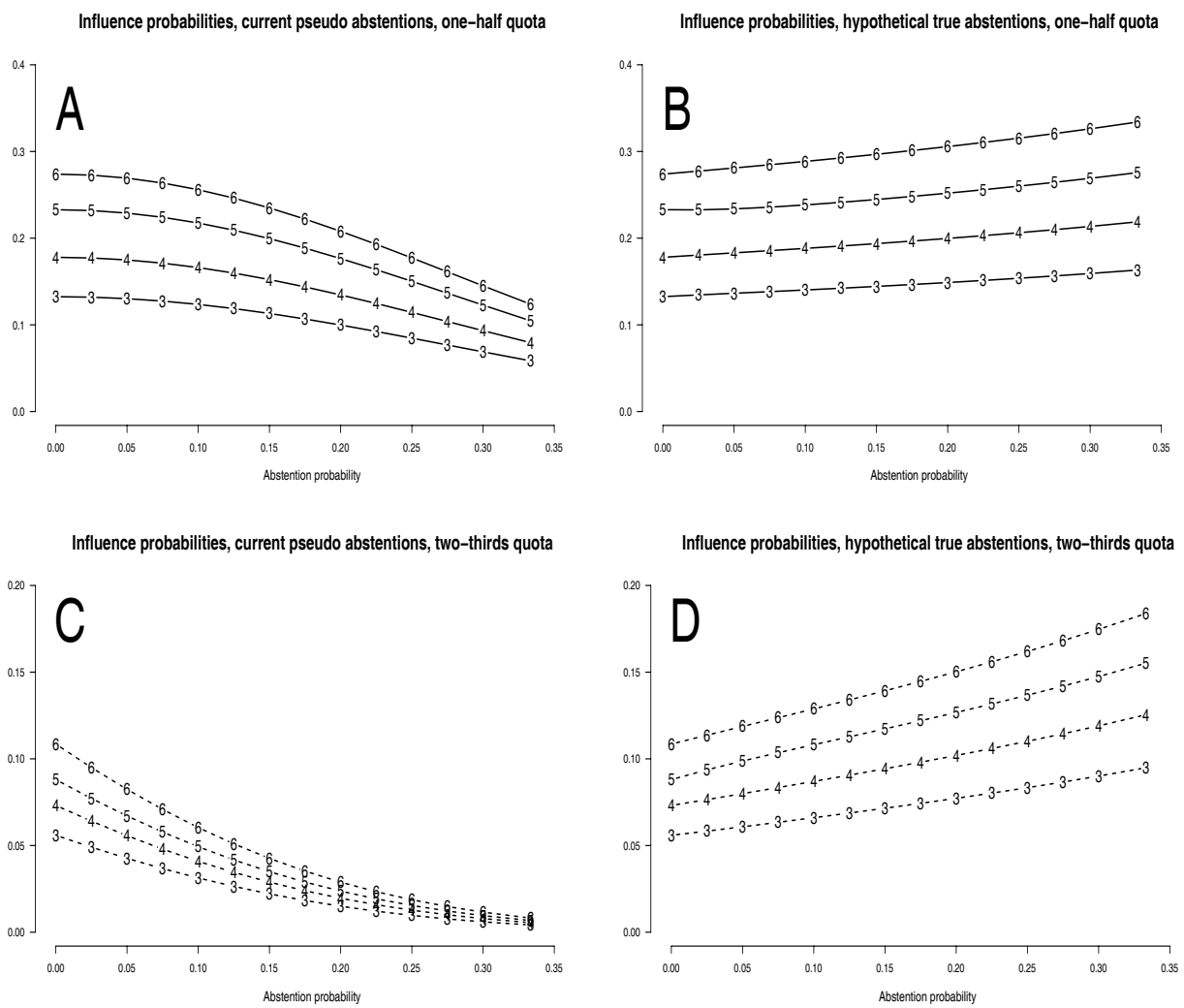

Figure 2.2 Influence probabilities of the States. Panels A and B apply to the one-half quota. The influence probabilities of all States decrease under current pseudo abstentions (A), and mildly increase under hypothetical true abstentions (B). The effect stands out more clearly for the two-thirds quota (C and D).

Figure 2.2 corroborates Schäuble's view as far as the influence probabilities and the power shares of the States are concerned. The panels show how the influence probabilities of the States change, as the abstention probability grows from 0 to $1 / 3$. The four lines refer to the States with voting weights $6,5,4,3$, from top to bottom. 
The upper panels apply to the one-half quota. Panel A, for pseudo abstentions, has all influence probabilities decreasing. Panel B treats true abstentions, and has all influence probabilities slightly increasing.

The lower panels are for the two-thirds quota. Panel C shows pseudo abstentions. The decrease of the influence probabilities is more pronounced, and falls almost down to zero. Panel D refers to true abstentions. The influence probabilities increase more than in Panel B.

Yet another index is provided by the power shares of the States, the quotients of the influence probability of a single State over the influence sensitivity of the decision rule. We find that all States acquire power shares that stay practically constant on the same level. In other words, no State gains or loses power relative to another State when switching from pseudo abstentions to true abstentions. The graphical message inherent in constant lines is rather uninformative, and hence omitted.

It is open to debate which conclusions to draw from the results shown. For instance, true abstentions and the two-thirds quota entail decision-making efficiencies that grow with the abstention probability, see Figure 2.1, right panel, lower line. Thus a higher abstention probability makes it increasingly easier to pass a constitutional amendment. This may not be a reasonable political goal. Instead one might contemplate maintaining the status quo of pseudo abstentions for the two-thirds quota, and to introduce true abstentions only when the normal business one-half quota is used.

In any case the example illustrates how the discussion of characteristic indices of decision rules may contribute to a design of sound procedures. The remaining sections turn to a general theory for the ternary setting.

\section{Ternary voting profiles and decision rules}

Let $N$ be a finite set, the assembly, of $n$ voters. A voting profile is a vector $a=\left(a_{j}\right)_{j \in N}$ with ternary components, $a_{j}:=1$ in case voter $j \in N$ is a Yea-voter, $a_{j}:=-1$ in case $j$ is a Nay-voter, and $a_{j}:=0$ in case voter $j$ abstains. Together the voting profiles form the ternary profile space

$$
\Omega_{N}:=\{-1,0,1\}^{N}
$$

Let $l_{N}:=(1, \ldots, 1)$ denote the unity vector with $n$ components, signifying the profile with everybody voting Yea. For a given voting profile $a \in \Omega_{N}$ the componentwise partial ordering $\leq$ of vectors induces the interval region of increasing consent, $\left[a, 1_{N}\right]:=\left\{b \in \Omega_{N}: a \leq b \leq 1_{N}\right\}$. A ternary decision rule is a subset $W_{N} \subseteq \Omega_{N}$ enjoying three properties:

$$
\begin{aligned}
& {\left[a, 1_{N}\right] \subseteq W_{N} \quad \text { for all } a \in W_{N},} \\
& 1_{N} \in W_{N}, \\
& 0 \notin W_{N} .
\end{aligned}
$$

The monotonicity property (3.1) is central: If a voting profile $a$ is in $W_{N}$ and $b$ exhibits at least as much consent as $a, b \geq a$, then $b$ is also in $W_{N}$. In view of (3.1), property (3.2) means that the decision rule $W_{N}$ is nonempty. Property (3.3) implies that profiles without 
a single Yea-voter are necessarily losing. The voting profiles in $W_{N}$ are termed positive or winning, for the reason that they are taken to represent the positive outcomes of the voting procedure. They are also known as winning coalitions, or winning configurations. The voting profiles in the complement $W_{N}^{c}:=\Omega_{N} \backslash W_{N}$ are called negative or losing.

Instead of our property (3.3) Felsenthal and Machover (1998, page 283) demand

$$
1_{N} \notin W_{N} .
$$

Under (3.4) a voting profile consisting of abstentions only and even including Nay-votes could still come out to be positive. We believe that such generosity is impractical and, therefore, adopt our more restrictive property (3.3).

A family of decision rules that is of particular relevance is formed by weighted decision rules. Let $w=\left(w_{j}\right)_{j \in N} \in(0, \infty)^{N}$ be a vector of voting weights. The sum of all components of $w$ is denoted by $w_{+}:=\sum_{j \in N} w_{j}$. For a given voting profile $a$ the sum of the voting weights of its Yea-voters is $\sum_{j: a_{j}=1} w_{j}$, and defines the profile weight of $a$.

The definition of weighted decision rules is more involved for ternary decision rules than for binary decision rules. The underlying idea is the same: the profile weight should exceed a certain prespecified quota. For the ternary case, however, the reference set for the quota is variable. For this reason we prefer to work with relative quotas $q \in[0,1)$. The relative quota $q$ is evaluated relative to the sum of the weights of the voters who vote Yea or Nay, excluding the weights of the voters who abstain.

To this end we define the indicator vector of the voters who vote either Yea or Nay, $|a|:=\left(\left|a_{j}\right|\right)_{j \in N}$. The indicator vectors that characterize the Yea-voters, the Nay-voters, and the voters who abstain then take the form

$$
(|a|+a) / 2, \quad(|a|-a) / 2, \quad 1_{N}-|a| .
$$

Again we use a subscript "+" to indicate the sum of all components. The number of Yea-voters is given by $\left(|a|_{+}+a_{+}\right) / 2$, the number of Nay-voters is $\left(|a|_{+}-a_{+}\right) / 2$, and the number of those who abstain is $n-|a|_{+}$. In a profile $a$ the cumulative weight of the Yeaand Nay-voters is given by the scalar product of the indicator vector $|a|$ and the weight vector $w, w^{\prime}|a|:=\sum_{j:\left|a_{j}\right|=1} w_{j}$.

We are now in a position to formally introduce the weighted decision rule $W_{N}(q ; w)$. By definition, it contains the voting profiles for which the profile weight exceeds the share $q$ of the cumulative weight of the Yea- and Nay-voters,

$$
W_{N}(q ; w):=\left\{a \in \Omega_{N}: \sum_{j: a_{j}=1} w_{j}>q w^{\prime}|a|\right\} .
$$

In particular, voters who abstain drop out from calculating the absolute quota $Q(a):=$ $q w^{\prime}|a|$. The definition demands strict inequality in order to satisfy property (3.3), that the voting profile with everybody abstaining cannot be winning. Freixas and Zwicker (2009, page 430) point out, though, that the two-thirds quota is often taken to mean "two-thirds or more", as is the case in the German Bundesrat, rather than "strictly more than two-thirds", as we demand in our definition above. 
Important examples for weighted ternary decision rules are the unanimity rule $U_{N}$ and the straight majority rule $M_{N}$ :

$$
\begin{aligned}
U_{N} & :=W_{N}\left(1-1 / n ; 1_{N}\right)=\left\{a \in \Omega_{N}: a_{+}=|a|_{+}>0\right\}, \\
M_{N} & :=W_{N}\left(1 / 2 ; 1_{N}\right)=\left\{a \in \Omega_{N}: a_{+}>0\right\} .
\end{aligned}
$$

The voting profiles where participant $j \in N$ is critical form an event of particular interest. Given a voting profile $a$, a voter $j$ is upgrade-critical when the profile $a$ is negative, but an increase in the degree of consent of $j$ turns it positive (by voter $j$ switching from Nay to abstention, or from Nay to Yea, or from abstention to Yea). Similarly voter $j$ is called downgrade-critical in $a$ when the profile $a$ starts out to be positive, but becomes negative as soon as the approval level of $j$ decreases (from Yea to abstention, or from Yea to Nay, or from abstention to Nay). A voter $j$ is critical or decisive when $j$ is upgrade- or downgrade-critical.

For a concise definition of critical events we introduce $\Pi_{N \backslash\{j\}}$, the projection of the profile space $\Omega_{N}=\{-1,0,1\}^{N}$ onto $\Omega_{N \backslash\{j\}}=\{-1,0,1\}^{N \backslash\{j\}}$, the $(n-1)$-dimensional marginal space omitting voter $j$. Given a marginal voting profile $b \in \Omega_{N \backslash\{j\}}$ without voter $j$, we introduce the full voting profiles $(b ; 1)$ with $j$ joining by voting Yea, $(b ; 0)$ with $j$ abstaining, and $(b ;-1)$ with $j$ voting Nay. The set of voting profiles where voter $j$ is critical takes the form

$$
C_{j}\left(W_{N}\right):=\left\{a \in \Omega_{N}:\left(\Pi_{N \backslash\{j\}}(a) ; 1\right) \in W_{N} \text { and }\left(\Pi_{N \backslash\{j\}}(a) ;-1\right) \in W_{N}^{c}\right\} .
$$

Let $D_{j}\left(W_{N}\right):=\Pi_{N \backslash\{j\}}\left(C_{j}\left(W_{N}\right)\right)$ be the image under the projection $\Pi_{N \backslash\{j\}}$ of the critical event $C_{j}\left(W_{N}\right)$. Then $C_{j}\left(W_{N}\right)$ is the preimage of $D_{j}\left(W_{N}\right)$ under the projection $\Pi_{N \backslash\{j\}}$,

$$
C_{j}\left(W_{N}\right)=\Pi_{N \backslash\{j\}}^{-1}\left(D_{j}\left(W_{N}\right)\right) .
$$

Next we turn to evaluating the events of interest by means of appropriate probability distributions.

\section{Ternary probability models}

Let $P$ be a probability distribution on the ternary profile space $\Omega_{N}$, and let $W_{N}$ be a ternary decision rule. The share of all positive voting profiles $P\left(W_{N}\right)$ is called the efficiency of $W_{N}$ under $P$. It serves as an indicator for the decision-making ability of the decision rule $W_{N}$ under $P$.

The influence probability of voter $j$ in the decision rule $W_{N}$ is defined to be the probability of $j$ being critical, $P\left(C_{j}\left(W_{N}\right)\right)$. The sum of all influence probabilities is termed the decision rule's influence sensitivity, $\Sigma_{P}\left(W_{N}\right):=\sum_{j \in N} P\left(C_{j}\left(W_{N}\right)\right)$.

The critical events $C_{j}\left(W_{N}\right), j \in N$, generally neither cover the profile space $\Omega_{N}$, nor are they pairwise disjoint. Hence there is no reason for the influence sensitivity to be equal to unity. However, the influence sensitivity can be used to normalize the influence probability of $j$ into $P\left(C_{j}\left(W_{N}\right)\right) / \Sigma_{P}\left(W_{N}\right)$, the power share of voter $j$ under $P$. The power shares form a probability distribution on the set of voters, $N$, preserving for any 
two voters $i \neq j$ the ratio of their influence probabilities, $P\left(C_{i}\left(W_{N}\right)\right) / P\left(C_{j}\left(W_{N}\right)\right)$. We shall not shift references spaces, though, and stick to the profile space $\Omega_{N}$.

In summary, efficiency and influence sensitivity are indices that refer to the decision rule $W_{N}$ as a whole, while influence probabilities and power shares describe characteristics of individual voters $j \in N$.

Two structural properties become essential for singling out particularly meaningful probability assumptions. A distribution $P$ is called selfdual when $P(\{a\})=P(\{-a\})$ holds for all voting profiles $a \in \Omega_{N}$. Selfduality means that the probability of a profile $a$ occurring for some proposal, equals the probability that the dual voting profile $-a$ emerges for the negated proposal.

A distribution $P$ is called permutationally invariant when $P \circ \pi^{-1}=P$ holds for all permutations $\pi$ of the assembly $N$. That is, it does not matter whether a profile $a$ lists voters in one way or another. To see the effect of permutational invariance, we write the profile space $\Omega_{N}$ as the disjoint union of the sets of voting profiles with fixed numbers $k$ of Yea-voters and $\ell$ of Nay-voters (and hence $n-k-\ell$ abstentions):

$$
\begin{aligned}
\Omega_{N} & =\biguplus_{k=0}^{n} \biguplus_{\ell=0}^{n-k}\left\{\begin{array}{c}
N \\
k ; \ell
\end{array}\right\}, \\
\left\{\begin{array}{c}
N \\
k ; \ell
\end{array}\right\} & :=\left\{a \in \Omega_{N}:\left(|a|_{+}+a_{+}\right) / 2=k \text { and }\left(|a|_{+}-a_{+}\right) / 2=\ell\right\} .
\end{aligned}
$$

The cardinality of the latter set is given by the trinomial coefficient

$$
\left(\begin{array}{c}
n \\
k ; \ell
\end{array}\right):=\left(\begin{array}{l}
n \\
k
\end{array}\right)\left(\begin{array}{c}
n-k \\
\ell
\end{array}\right)=\frac{n !}{k ! \ell !(n-k-\ell) !} .
$$

Within such a profile subset, a permutationally invariant distribution behaves like a uniform distribution:

$$
P(\{a\})=\frac{1}{\left(\begin{array}{c}
n \\
k ; \ell
\end{array}\right)} P\left(\left\{\begin{array}{c}
N \\
k ; \ell
\end{array}\right\}\right) \quad \text { for all } a \in\left\{\begin{array}{c}
N \\
k ; \ell
\end{array}\right\} .
$$

A voting profile $a \in \Omega_{N}$ is a success for voter $j$, and voter $j$ is said to agree with the profile $a$, when the profile is positive and $j$ votes Yea $\left(a \in W_{N}, a_{j}=1\right)$, or when the profile is negative and $j$ votes Nay $\left(a \in W_{N}^{c}, a_{j}=-1\right)$. A positive voting profile is a failure for a Nay-voter, as is a negative voting profile for a Yea-voter. An abstaining voter $j$ is taken to be indifferent, whence the profile is counted neither as a success nor as a failure.

The success margin $\sigma_{W_{N}}(a)$ of the voting profile $a$ is of particular interest. It is defined as the difference between the number of voters for which a voting profile is a success, and the number of the voters for which it is a failure:

$$
\sigma_{W_{N}}(a):=\left\{\begin{aligned}
a_{+} & \text {in case } a \in W_{N}, \\
-a_{+} & \text {in case } a \in W_{N}^{c}
\end{aligned}\right.
$$


A voting profile $a \in W_{N}$ that is positive and at the same time constitutes a failure to a majority of the voters has a success margin that is negative. Hence the negative part of the success margin is called the majority deficit, $\delta_{W_{N}}(a):=\sigma_{W_{N}}^{-}(a)=\left(\left|\sigma_{W_{N}}(a)\right|-\right.$ $\left.\sigma_{W_{N}}(a)\right) / 2$. The expected success margin of an arbitrary decision rule $W_{N}$ is bounded by the expected success margins of the straight majority rule and the unanimity rule, as follows.

Proposition 4.1 Let $W_{N}$ be a ternary decision rule for an assembly $N$.

(i) The success margin $\sigma_{W_{N}}$ and the majority deficit $\delta_{W_{N}}$ satisfy $\sigma_{W_{N}}=\sigma_{M_{N}}-2 \delta_{W_{N}} \leq$ $\sigma_{M_{N}}$, where $\sigma_{M_{N}}$ is the success margin of the straight majority rule $M_{N}$. In particular, every distribution $P$ fulfills

$$
\mathrm{E}_{P}\left[\sigma_{W_{N}}\right] \leq \mathrm{E}_{P}\left[\sigma_{M_{N}}\right]
$$

(ii) Every selfdual distribution $P$ fulfills

$$
\mathrm{E}_{P}\left[\sigma_{W_{N}}\right]=2 \sum_{a \in W_{N}} a_{+} P(\{a\})=2 \sum_{k=1}^{n} \sum_{\ell=0}^{n-k}(k-\ell) P\left(W_{N} \cap\left\{\begin{array}{c}
N \\
k ; \ell
\end{array}\right\}\right) .
$$

(iii) Every selfdual and permutationally invariant distribution $P$ fulfills

$$
\mathrm{E}_{P}\left[\sigma_{W_{N}}\right] \geq \mathrm{E}_{P}\left[\sigma_{U_{N}}\right]
$$

Proof: (i) The absolute value of any success margin is equal to the success margin of the straight majority rule, since $\left|\sigma_{W_{N}}(a)\right|=\left|a_{+}\right|=\sigma_{M_{N}}(a)$. The assertions follow from $\delta_{W_{N}}=\left(\sigma_{M_{N}}-\sigma_{W_{N}}\right) / 2$, and $\sigma_{W_{N}} \leq\left|\sigma_{W_{N}}\right|=\sigma_{M_{N}}$.

(ii) With the indicator function $\mathbb{1}\left\{a \in W_{N}\right\}=1$ in case $a \in W_{N}$ and $\mathbb{1}\left\{a \in W_{N}\right\}=0$ in case $a \in W_{N}^{c}$, the success margin becomes $\sigma_{W_{N}}(a)=\left(2 \cdot \mathbb{1}\left\{a \in W_{N}\right\}-1\right) a_{+}$. We obtain $\mathrm{E}_{P}\left[\sigma_{W_{N}}\right]=2 \sum_{a \in W_{N}} a_{+} P(\{a\})-\sum_{a \in \Omega_{N}} a_{+} P(\{a\})$. The last sum vanishes due to $a_{+}=-\left(-a_{+}\right)$and the selfduality of $P$ :

$$
2 \sum_{a \in \Omega_{N}} a_{+} P(\{a\})=\sum_{a \in \Omega_{N}} a_{+} P(\{a\})-\sum_{a \in \Omega_{N}}(-a)_{+} P(\{-a\})=0 .
$$

In view of $a_{+}=k-\ell$ the second equality is a rearrangement according to the number of Yea-voters, $k=\left(|a|_{+}+a_{+}\right) / 2$, and the number of Nay-voters, $\ell=\left(|a|_{+}-a_{+}\right) / 2$. In the first sum $k$ ranges from 1 to $n$ since $0 \notin W_{N}$ and hence $\left\{\begin{array}{c}N \\ 0 ; \ell\end{array}\right\} \notin W_{N}$, due to the monotonicity condition (3.1).

(iii) Since for every voting profile $a \in\left\{\begin{array}{c}N \\ k ; \ell\end{array}\right\}$ its dual voting profile $-a$ is in $\left\{\begin{array}{c}N \\ \ell ; k\end{array}\right\}$, selfdual distributions satisfy $P\left(\left\{\begin{array}{c}N \\ k ; \ell\end{array}\right\}\right)=P\left(\left\{\begin{array}{c}N \\ \ell ; k\end{array}\right\}\right)$. Permutational invariance yields

$$
P(\{a\})=P(\{b\})=\frac{1}{\left(\begin{array}{c}
n \\
k ; \ell
\end{array}\right)} P\left(\left\{\begin{array}{c}
N \\
k ; \ell
\end{array}\right\}\right) \quad \text { for all } a \in\left\{\begin{array}{c}
N \\
k ; \ell
\end{array}\right\} \text { and } b \in\left\{\begin{array}{c}
N \\
\ell ; k
\end{array}\right\}
$$


For the unanimity rule $U_{N}=\biguplus_{k=1}^{n}\left\{\begin{array}{c}N \\ k ; 0\end{array}\right\}$ part (ii) entails

$$
\mathrm{E}_{P}\left[\sigma_{W_{N}}\right]=\mathrm{E}_{P}\left[\sigma_{U_{N}}\right]+2 \sum_{k=1}^{n} \sum_{\ell=1}^{n-k}(k-\ell) P\left(W_{N} \cap\left\{\begin{array}{c}
N \\
k ; \ell
\end{array}\right\}\right) .
$$

We show that the double sum is nonnegative. Note that the summand vanishes for $\ell=k$. Omitting this case and applying permutational invariance and selfduality, we obtain

$$
\begin{aligned}
& \sum_{k=1}^{n} \sum_{\ell=1}^{k-1}(k-\ell) P\left(W_{N} \cap\left\{\begin{array}{c}
N \\
k ; \ell
\end{array}\right\}\right)+\sum_{k=1}^{n} \sum_{\ell=k+1}^{n-k}(k-\ell) P\left(W_{N} \cap\left\{\begin{array}{c}
N \\
k ; \ell
\end{array}\right\}\right) \\
& =\sum_{k=2}^{n} \sum_{\ell=1}^{k-1}(k-\ell)\left(P\left(W_{N} \cap\left\{\begin{array}{c}
N \\
k ; \ell
\end{array}\right\}\right)-P\left(W_{N} \cap\left\{\begin{array}{c}
N \\
\ell ; k
\end{array}\right\}\right)\right) \\
& =\sum_{k=2}^{n} \sum_{\ell=1}^{k-1} \frac{(k-\ell)}{\left(\begin{array}{c}
n \\
k ; \ell
\end{array}\right)}\left(\#\left(W_{N} \cap\left\{\begin{array}{c}
N \\
k ; \ell
\end{array}\right\}\right)-\#\left(W_{N} \cap\left\{\begin{array}{c}
N \\
\ell ; k
\end{array}\right\}\right)\right) .
\end{aligned}
$$

Since a decision rule $W_{N}$ is monotone, by its defining property (1), any set of positive voting profiles with many Yea-voters $(k)$ outnumbers any set of positive voting profiles with fewer Yea-voters $(l)$. Hence the final expression is nonnegative.

\section{The ternary Penrose-Banzhaf model}

The ternary Penrose-Banzhaf model assumes that all voters act independently and that the individual marginal distributions are Multinomial $((1-t) / 2, t,(1-t) / 2)$. Thus voters are taken to share a common abstention probability $t \in[0,1)$, and to vote Yea or Nay with half of the remaining probability, $(1-t) / 2$. Hence the ternary Penrose-Banzhaf distribution $P_{N}^{t}$, contingent upon the "ternary" parameter $t$, is defined through

$$
P_{N}^{t}(\{a\}):=t^{n-|a|_{+}}\left(\frac{1-t}{2}\right)^{|a|_{+}} \quad \text { for all } a \in \Omega_{N} .
$$

It is easy to see that these distributions are selfdual and permutationally invariant. They are also projectively consistent in the sense that the identity $P_{N}^{t} \circ \Pi_{N \backslash\{j\}}^{-1}=P_{N \backslash\{j\}}^{t}$ holds for all $j \in N$.

Proposition 5.1 looks at the sets of voting profiles that are a success for voter $j$ (that is, where $j$ agrees with the outcome), or a failure:

$$
\begin{gathered}
A_{j}\left(W_{N}\right):=\left\{a \in \Omega_{N}: a \in W_{N} \text { and } a_{j}=1, \text { or } a \in W_{N}^{c} \text { and } a_{j}=-1\right\}, \\
F_{j}\left(W_{N}\right):=\left\{a \in \Omega_{N}: a \in W_{N} \text { and } a_{j}=-1, \text { or } a \in W_{N}^{c} \text { and } a_{j}=1\right\} .
\end{gathered}
$$

We call $P_{N}^{t}\left(A_{j}\left(W_{N}\right)\right)$ the success probability and $P_{N}^{t}\left(F_{j}\left(W_{N}\right)\right)$ the failure probability of voter $j$. They are related to the influence probability of $j$, as follows. 
Proposition 5.1 Let $W_{N}$ be a ternary decision rule for an assembly $N$. The ternary Penrose-Banzhaf success, failure, and influence probabilities for voter $j \in N$ fulfill

$$
\begin{aligned}
& P_{N}^{t}\left(A_{j}\left(W_{N}\right)\right)=\frac{1-t}{2}+\frac{1-t}{2} P_{N}^{t}\left(C_{j}\left(W_{N}\right)\right), \\
& P_{N}^{t}\left(F_{j}\left(W_{N}\right)\right)=\frac{1-t}{2}-\frac{1-t}{2} P_{N}^{t}\left(C_{j}\left(W_{N}\right)\right) .
\end{aligned}
$$

Proof: Voting profiles in which voter $j$ abstains are neither a success nor a failure. In the complementary profiles $j$ votes Yea or Nay, $\left\{a_{j}= \pm 1\right\}:=\left\{a \in \Omega_{N}: a_{j} \neq 0\right\}$. Clearly we have $A_{j}\left(W_{N}\right)=A_{j}\left(W_{N}\right) \cap\left\{a_{j}= \pm 1\right\}$.

Every voting profile in which voter $j$ 's Yea or Nay is critical is a success for $j$. This first case occurs with probability

$$
P_{N}^{t}\left(C_{j}\left(W_{N}\right) \cap\left\{a_{j}= \pm 1\right\}\right)=P_{\{j\}}^{t}(\{ \pm 1\}) P_{N \backslash\{j\}}^{t}\left(D_{j}\left(W_{N}\right)\right)=(1-t) P_{N}^{t}\left(C_{j}\left(W_{N}\right)\right) .
$$

For a voting profile $a$ in which voter $j$ 's Yea or Nay is not critical, either $(b ;-1)$ or $(b ; 1)$ is a success for $j$, where the projection $b=\Pi_{N \backslash\{j\}}(a)$ comprises the votes of the others. Since the two possibilities are equally likely, the contribution of the second case is

$$
\frac{1}{2} P_{N}^{t}\left(C_{j}\left(W_{N}\right)^{c} \cap\left\{a_{j}= \pm 1\right\}\right)=\frac{1-t}{2}\left(1-P_{N}^{t}\left(C_{j}\left(W_{N}\right)\right)\right) .
$$

The sum of the two cases yields the success probability of $j$ as given in the assertion. The failure probability formula follows from the identity $P_{N}^{t}\left(A_{j}\left(W_{N}\right)\right)+P_{N}^{t}\left(F_{j}\left(W_{N}\right)\right)=$ $P_{N}^{t}\left(\left\{a_{j}= \pm 1\right\}\right)=1-t$.

We are now in a position to show that the ternary Penrose-Banzhaf influence sensitivity coincides with the Penrose-Banzhaf mean success margin, up to a scaling constant that solely depends on the abstention probability $t$.

Proposition 5.2 Let $W_{N}$ be a ternary decision rule for an assembly $N$. Under the ternary Penrose-Banzhaf distribution with abstention probability $t \in[0,1)$, the influence sensitivity of $W_{N}$ and the mean success margin of $W_{N}$ fulfill

$$
\Sigma_{P_{N}^{t}}\left(W_{N}\right)=\frac{1}{1-t} \mathrm{E}_{P_{N}^{t}}\left[\sigma_{W_{N}}\right]
$$

Proof: For every voter $j$ we define the success function

$$
\sigma_{j}(a):=\left\{\begin{aligned}
1 & \text { in case } a \in A_{j}\left(W_{N}\right), \\
0 & \text { in case } a_{j}=0, \\
-1 & \text { in case } a \in F_{j}\left(W_{N}\right) .
\end{aligned}\right.
$$

The success margin is the sum of the individual success functions, $\sigma_{W_{N}}(a)=\sum_{j \in N} \sigma_{j}(a)$. From Proposition 5.1 we obtain

$$
\mathrm{E}_{P_{N}^{t}}\left[\sigma_{W_{N}}\right]=\sum_{j \in N}\left(P_{N}^{t}\left(A_{j}\left(W_{N}\right)\right)-P_{N}^{t}\left(F_{j}\left(W_{N}\right)\right)\right)=(1-t) \Sigma_{P_{N}^{t}}\left(W_{N}\right) .
$$


Propositions 4.1 and 5.2 provide bounds for the ternary Penrose-Banzhaf influence sensitivity $\Sigma_{P_{N}^{t}}\left(W_{N}\right)$ of an arbitrary ternary decision rule $W_{N}$. The lower bound is given by the unanimity rule, through $\Sigma_{P_{N}^{t}}\left(U_{N}\right)=n\left(\frac{1+t}{2}\right)^{n-1}$. The upper bound stems from the straight majority rule,

$$
\Sigma_{P_{N}^{t}}\left(M_{N}\right)=\sum_{k=1}^{n} \sum_{\ell=0}^{k-1}(k-\ell)\left(\begin{array}{c}
n \\
k ; \ell
\end{array}\right) t^{n-k-\ell}\left(\frac{1-t}{2}\right)^{k+\ell-1} .
$$

\section{Discussion}

The present paper proposes a probabilistic approach to ternary decision rules that permit committee members to vote Yea or Nay or to abstain. Our motivation to calculate some characteristic indices for such rules arises from the political debate in Germany to improve the decision procedures used in the Bundesrat, in order to increase the Bundesrat's decision-making efficiency.

Current Bundesrat procedures implement pseudo abstentions, as described in Section 2, with an abstention factually being viewed as just another way of voting Nay. Such a system is modeled by a distribution wherein voters act independently, and vote Yea with probability $(1-t) / 2$ and Nay with the complementary probability $(1+t) / 2$. Thus the binary voting profile $a \in\{-1,1\}^{N}$ occurs with probability

$$
\left(\frac{1-t}{2}\right)^{\left(n+a_{+}\right) / 2}\left(\frac{1+t}{2}\right)^{\left(n-a_{+}\right) / 2}
$$

The pseudo abstention curves in Figures 2.1 and 2.2 were calculated with these probabilities, using generating functions as in Bilbao et al. (2000) and Leech (2002) for efficient computation. The example is interesting also from the theoretical viewpoint, in that it is based on a probability distribution that fails to be selfdual but otherwise looks quite reasonable.

True abstentions generate ternary voting profiles $a \in\{-1,0,1\}^{N}$, with the additional code $a_{j}=0$ signaling an abstention of voter $j$. A pertinent probability model is obtained by extending the binary Penrose-Banzhaf model to the ternary situation. The ternary Penrose-Banzhaf distribution comes with a "ternary" parameter $t$, the probability with which voters abstain. The model assumes the abstention probability $t$ to be the same for all voters, and hence assigns to the ternary voting profile $a \in\{-1,0,1\}^{N}$ the probability

$$
t^{n-|a|_{+}}\left(\frac{1-t}{2}\right)^{|a|_{+}} \text {. }
$$

The true abstention curves in Figures 2.1 and 2.2 were calculated under the ternary Penrose-Banzhaf model.

Our Propositions 5.1 and 5.2 reproduce the results of Felsenthal and Machover (1998, Section 8.3) for the special case $t=1 / 3$, that is, when a Yea, a Nay, and an abstention are equally likely. We believe that $1 / 3$ gets close to the maximum of an abstention 
probability to be met in practice. For this reason the curves in Figures 2.1 and 2.2 do not extend beyond $1 / 3$.

The ternary Penrose-Banzhaf model includes a vanishing abstention probability, $t=0$. In this case, ternary decision rules degenerate to binary decision rules. Accordingly, the present formulas simplify to those familiar from binary decision rules, see Felsenthal and Machover (1998, Section 3.2) and Ruff and Pukelsheim (2010).

There is a more sophisticated way to extract binary decision rules from the ternary case, by viewing the binary profile space $\{-1,1\}^{N}$ as a subset of the ternary profile space $\{-1,0,1\}^{N}$. Indeed, every ternary decision rule $W_{N} \subset\{-1,0,1\}^{N}$ has a binary trace $B\left(W_{N}\right):=W_{N} \cap\{-1,1\}^{N}$ that turns out to be a well-defined binary decision rule, in that it fulfills properties (3.1), (3.2), and (3.4) of Section 3.

Furthermore, a probability distribution $P$ on the ternary profile space induces a distribution on the binary profile space through conditioning,

$$
P\left(\{a\} \mid\{-1,1\}^{N}\right)=\frac{P(\{a\})}{P\left(\{-1,1\}^{N}\right)},
$$

for all binary voting profiles $a \in\{-1,1\}^{N}$. Not surprisingly, all ternary Penrose-Banzhaf distributions induce the same binary trace, namely the well-known binary PenroseBanzhaf distribution.

In summary, our results on the ternary Penrose-Banzhaf model afford a passage back to the binary Penrose-Banzhaf model not only through the singular parameter choice $t=0$, but also, by conditioning, for all conceivable parameter values $t \in[0,1)$.

Acknowledgments. We are grateful to Moshé Machover, Antony Unwin, Bill Zwicker, and two referees for critical comments on earlier versions of the paper.

\section{References}

[1] Bilbao, J. M., Fernández, J. R., Jiménez Losada, A., López, J. J. (2000). Generating functions for computing power indices efficiently. Top 8 191-213.

[2] Bolger, E. M. (1983). The Banzhaf index for multicandidate presidential elections. SIAM Journal on Algebraic and Discrete Methods 4 442-458.

[3] Bolger, E. M. (2002). Characterizations of two power indices for voting games with $r$ alternatives. Social Choice and Welfare 19 709-721.

[4] Braham, M., Steffen, F. (2002). Voting power in games with abstentions. In: Holler, M. et al. (Editors), Power and Fairness, Jahrbuch für Neue Politische Ökonomie 20, Mohr-Siebeck, 333-348.

[5] Côrte-Real, P., Pereira, P. (2004). The voter who wasn't there: Referenda, representation and abstention. Social Choice and Welfare 22 349-369. 
[6] Felsenthal, D. S., Machover, M. (1997). Ternary voting games. International Journal of Game Theory 26 335-351.

[7] Felsenthal, D. S., Machover, M. (1998). The Measurement of Voting Power. Edward Elgar, Cheltenham.

[8] Felsenthal, D. S., Machover, M. (2001). Models and reality: The curious case of the absent abstention. In: Holler, M., Owen, G. (Editors), Power Indices and Coalition Formation, Kluwer, 87-103.

[9] Felsenthal, D. S., Machover, M. (2005). Voting power measurement: A story of misreinvention. Social Choice and Welfare 25 485-506.

[10] Fishburn, P. C. (1973). The Theory of Social Choice. Princeton University Press.

[11] Freixas, J. (2005a). Banzhaf measures for games with several levels of approval in the input and output. Annals of Operations Research 137 45-66.

[12] Freixas, J. (2005b). The Shapley-Shubik power index for games with several levels of approval in the input and output. Decision Support Systems 39 185-195.

[13] Freixas, J., Palacios, D. (2010). Probabilistic power indices for voting rules with abstention. Paper presented at the Computational Foundations of Social Choice Seminar, Dagstuhl, 7-12 March 2010.

[14] Freixas, J., Zwicker, W. S. (2003). Weighted voting, abstention, and multiple levels of approval. Social Choice and Welfare 21 399-431.

[15] Freixas, J., Zwicker, W. S. (2009). Anonymous yes-no voting with abstention and multiple levels of approval. Games and Economic Behavior 67 428-444.

[16] Laruelle, A., Valenciano, F. (2005). Assessing success and decisiveness in voting situations. Social Choice and Welfare 24 171-197.

[17] Laruelle, A., Valenciano, F. (2010a). Quaternary dichotomous voting rules. University of the Basque Country, Departamento de Fundamentos del Análisis Económico I, Working Paper Series II, No. 41/10.

[18] Laruelle, A., Valenciano, F. (2010b). Majorities with a quorum. University of the Basque Country, Departamento de Fundamentos del Análisis Económico I, Working Paper Series II, No. 42/10.

[19] Leech, D. (2002). Computation of Power Indices. Warwick Economic Research Papers No 644, Department of Economics, University of Warwick.

[20] Lindner, I. (2004). Power Measures in Large Weighted Voting Games. PhD Thesis, Universität Hamburg.

[21] Reinhardt, V. (2006). Geschichte der Schweiz. Beck, München.

[22] Rubinstein, A. (1980). Stability of decision systems under majority rule. Journal of Economic Theory 23 150-159. 
[23] Ruff, O., Pukelsheim, F. (2010). A probabilistic synopsis of binary decision rules. Social Choice and Welfare 35 501-516.

[24] Straffin, P. (1988). The Shapley-Shubik and Banzhaf power indices as probabilities. In: Roth, A. E. (Editor), The Shapley Value. Essays in Honor of Lloyd S. Shapley, Cambridge University Press, 71-81.

Olga Birkmeier

Institute for Mathematics

University of Augsburg

86135 Augsburg

Germany

olga.birkmeier@math.uni-augsburg.de
Andreas Käufl

Institute for Mathematics

University of Augsburg

86135 Augsburg

Germany

andreas.kaeufl@googlemail.com

Friedrich Pukelsheim

Institute for Mathematics

University of Augsburg

86135 Augsburg

Germany

pukelsheim@math.uni-augsburg.de 